\title{
2018 Year in Review: Sleep
}

\author{
Brian W Carlin
}

\author{
Obstructive Sleep Apnea and Comorbid Conditions \\ Cardiovascular Comorbidities \\ Depression \\ Predicting Postoperative Complications \\ Obstructive Sleep Apnea: Testing and Technologies \\ Portable Sleep Testing \\ Consumer Sleep Technologies \\ Management of Obstructive Sleep Apnea \\ Weight Management \\ Positive Airway Pressure Management \\ Oral Appliance Therapy \\ Surgical Therapy \\ Pharmacologic Therapy \\ Exercise and Rehabilitation \\ Therapy Adherence \\ Hospital Readmissions and Adherence \\ Patient-Engagement Technologies \\ Other Publications \\ Medical Cannabis \\ Personalized Management \\ Reflections on Nasal CPAP and Obstructive Sleep Apnea \\ Summary
}

\begin{abstract}
Sleep-disordered breathing affects a significant portion of the population worldwide. It is associated with many comorbid conditions, including heart failure and depression. Advances in the field regarding the diagnosis and treatment of sleep-disordered breathing are occurring on an increasing basis. This review will discuss the latest findings in the field with an emphasis on people who have obstructive sleep apnea. [Respir Care 2019;64(8):1007-1013. () 2019 Daedalus Enterprises]
\end{abstract}

\section{Obstructive Sleep Apnea and Comorbid Conditions}

\section{Cardiovascular Comorbidities}

Cardiovascular comorbidities have a well-known association with obstructive sleep apnea (OSA). Investigators performed a study to determine whether the combined presence of COPD and severe OSA, as defined by the apnea hypopnea index (AHI) or degree of nocturnal desaturation, was associated with increased hazards of cardiovascular events and mortality. Subject data were prospectively collected from 1994 to 2010 for adults with
COPD and suspected OSA (ie, AHI $>30$ or $\geq 10 \mathrm{~min}$ of sleep time spent with oxygen saturation $<90 \%$ ) who underwent a polysomnogram (PSG); these subjects were compared to subjects who had a lower thresholds of AHI or desaturation time periods. ${ }^{1}$

Over 9.4 years, 10,149 subjects were included in this study. ${ }^{1}$ The primary outcome measure was a composite of hospitalization due to acute myocardial infarction, stroke, congestive heart failure, cardiac revascularization procedures, or death from any cause. A positive outcome was noted in $16.4 \%$ of subjects, with a greater hazard of out- 
come observed in those with COPD who spent at least 10 min of sleep with an oxygen saturation $<90 \%$ (heart rate $=1.91,95 \%$ CI $1.60-2.28$ ), but not with those who had an AHI $>30$. The highest hazard of outcome was associated with the coexistence of COPD and an AHI $>30$ in subjects who were not treated for OSA (heart rate $=2.01$, 95\% CI 1.55-2.62). The investigators concluded that, in adults with suspected OSA, the occurrence of nocturnal hypoxemia and COPD was associated with an increased hazard of cardiovascular events and mortality. A synergistic effect was found only in women.

\section{Depression}

Depression is another comorbid condition in people who have both OSA and cardiovascular disease. The effects of treatment for those with non-sleepy OSA and cardiovascular disease is unknown. Balcan et $\mathrm{al}^{2}$ performed a secondary analysis of data from the RICCADSA (Randomized Intervention with CPAP in CAD and OSA) trial conducted between 2005 and 2013 for subjects who had coronary artery disease and non-sleepy OSA (AHI $\geq 15 / \mathrm{h}$, Epworth Sleepiness Scale $>10$ at baseline). The impact of positive airway pressure (PAP) therapy on self-ratings of depression was studied.

Subjects were randomized to either CPAP therapy $(n=99)$ or no CPAP therapy $(n=104)$. The primary outcome measure was between-group difference in the absolute change in the self-reported depression score from baseline. No significant between-group differences were observed in the self-reported depression score during follow-up. The proportion of subjects with depression decreased from $30.3 \%$ at baseline to $16.2 \%$ after 3 months and to $13.1 \%$ after 12 months in the CPAP group, and from $25.0 \%$ at baseline to $23.1 \%$ after 3 months and to $24.0 \%$ after 12 months in the no CPAP group $(P=.001)$. An association between the duration of hours of CPAP usage per night and longitudinal decline in self-reported depression score was noted. CPAP usage, categorized as $3 \mathrm{~h}, 4 \mathrm{~h}$, or $5 \mathrm{~h}$ each night, was significantly associated

Dr Carlin is affiliated with Sleep Medicine and Lung Health Consultants, Ingomar, Pennsylvania.

Dr Carlin has disclosed relationships with Sunovion, Monaghan, Astra Zeneca, and Nonin.

Correspondence: Brian W Carlin MD FAARC, Sleep Medicine and Lung Health Consultants, PO Box 174, Ingomar, PA 15127. E-mail: bwcmd@yahoo.com.

Dr Carlin presented a version of this paper at the Year in Review of the AARC Congress 2018, held December 4-7, 2018 in Las Vegas, Nevada.

DOI: $10.4187 /$ respcare. 07063
Table 1. Factors Associated With Postoperative Complications in Patients With OSA

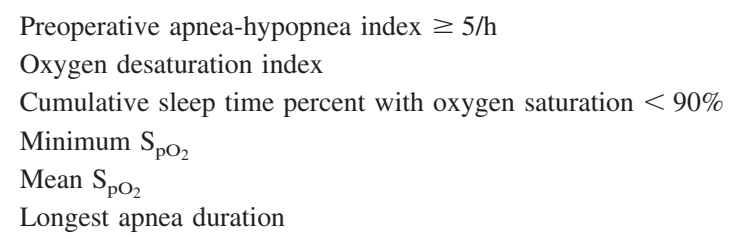

with an improvement in self-reported depression score (odds ratios $=3.92,4.45$, and 4.89 , respectively). It was concluded that for subjects with depression who have nonsleepy OSA and coronary artery disease, 3 months of CPAP treatment improved depression scores with the improvement in mood persisting for up to 12 months.

\section{Predicting Postoperative Complications}

OSA has been shown to be associated with an increased risk of postoperative complications. At present, however, risk stratification using OSA-associated parameters derived from PSG or nocturnal overnight oximetry to predict these postoperative complications has not been well established. Suen et $\mathrm{al}^{3}$ reviewed 21 articles to determine the association between postoperative adverse effects and parameters extracted from in-laboratory PSG, portable PSG, or overnight oximetry. The parameters that were associated with postoperative complications are listed in Table 1. The development of clinical pathways utilizing these sleep-study parameters may be helpful to reduce postoperative complications.

\section{OSA: Testing and Technologies}

\section{Portable Sleep Testing}

The use of portable testing for the diagnosis of OSA has increased over the last decade. A home sleep apnea test is an alternative to PSG for the diagnosis of uncomplicated OSA in adults with signs and symptoms indicating an increased risk of moderate to severe OSA. The American Academy of Sleep Medicine (AASM) recently published position paper on the clinical use of home sleep apnea test, stating that health care providers (eg, physicians, nurse practitioners, and physician's assistants) can diagnose medical conditions such as OSA and primary snoring. ${ }^{4}$ The need for, and the appropriate use of, home sleep apnea testing must be based on the individual's medical history and an in-person examination by a physician or advanced 
Table 2. Summary of the AASM Position Statement on HSAT

1. It is a medical assessment that must be ordered by a physician to diagnose OSA or evaluate treatment efficacy.

2. It should not be used for general screening of asymptomatic clinical populations.

3. Diagnosis, assessment treatment efficacy, and treatment decisions must not be based solely on automatically scored HSAT data.

4. The raw data from the HSAT device must be reviewed and interpreted by a physician who is either board-certified in sleep medicine or overseen by a board-certified sleep medicine physician.

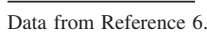

AASM $=$ American Academy of Sleep Medicine

HSAT $=$ home sleep apnea testing

OSA $=$ obstructive sleep apnea

practice provider. Additional positions on home sleep apnea test are shown in Table 2 .

Many studies comparing the results of home sleep apnea test and PSG in the out-patient setting have been conducted. However studies in the in-patient setting have been limited. Aurora et $\mathrm{al}^{5}$ examined whether portable sleep monitoring compared with complete PSG can accurately diagnose sleep apnea in subjects who were hospitalized with decompensated heart failure. The study included 53 subjects hospitalized with decompensated heart failure, who underwent both home sleep apnea testing and PSG. The PSGs were scored for both obstructive and central sleep-disordered breathing events in a blinded fashion. The correlation coefficient for the overall AHI for the 2 diagnostic methods was 0.94 (95\% CI 0.89-0.96). The average difference in the AHI between the 2 methods was 3.6 events/h. There was a strong concordance between both methods when analyzing central and obstructive event indexes. Complete agreement in the classification of the severity of sleep apnea between the 2 methods was seen in $89 \%$ of the sample. The investigators concluded that portable sleep monitoring can be helpful to accurately diagnose sleep apnea in hospitalized subjects with heart failure. This may ultimately help promote earlier initiation of treatment and improve overall outcomes for this particular patient population.

\section{Consumer Sleep Technologies}

Consumer sleep technologies are becoming more widely used to measure and to help improve sleep quality. There has been, however, a lack of validation with these technologies against the accepted standard PSG. In addition, these devices have not been cleared by the FDA for assessing sleep quality given the lack of scientific validation of these technologies. Thus, they should not be used for the diagnosis or treatment of sleep disorders at this time. An opportunity for the use of these devices does exist, however, regarding the enhancement of the patient-clinician interaction when presented in the context of an appropriate clinical evaluation. Future validation, access to raw data and algorithms, and FDA oversight are needed when considering these devices for either the diagnosis or response to treatment for patients with sleep-disordered breathing. 6

\section{Management of OSA}

\section{Weight Management}

An official American Thoracic Society clinical practice guideline regarding the role of weight management in the treatment of adult OSA was recently made available. The grading of recommendations assessment, development, and evaluation approach (GRADE) was used to evaluate the literature regarding the effects of weight loss (ie, behavioral, pharmacologic, and surgical treatments) on the effect of OSA severity, common comorbidity reversal, and quality of life. ${ }^{7}$ The guideline recommends that clinicians assess weight and incorporate and tailor weight-management strategies based on individual patient preferences. A strong recommendation was made that subjects who have OSA and are overweight or obese should be treated with comprehensive lifestyle interventions consisting of a reduced-calorie diet, exercise, and behavioral guidance. Pharmacologic therapy and bariatric surgical therapy are appropriate for select individuals who require further assistance with weight loss. Conditional recommendations were made for other management tools, including a reduced-calorie diet and exercise/increased physical activity.

\section{PAP Therapy}

PAP therapy is the mainstay of treatment for OSA. A multi-center trial ( $N=208$ subjects) with OSA syndrome was conducted to determine whether auto-CPAP was equivalent to fixed-CPAP in terms of improved long-term sleepiness. The subjects had a median average Epworth Sleepiness Scale score of 13 and an AHI of 48.4/h. Subjects were randomized to treatment with either auto-CPAP (range of 5-15 $\mathrm{cm} \mathrm{H}_{2} \mathrm{O}$ pressure support) or fixed CPAP. The fixed CPAP pressure was set at the 90th percentile applied by auto-CPAP during an adaptation trial lasting 2-4 weeks. ${ }^{8}$

After 2 years of therapy, in the intention-to-treat analysis, a similar reduction in sleepiness (compared to pretreatment baseline) was noted in subjects receiving autoCPAP. The 95\% CI of difference in Epworth Sleepiness Scale minus the reduction between auto-CPAP and fixed CPAP was -0.9 to +1.4 , and the $95 \% \mathrm{CI}$ of difference in increase in sleep-resistance time was -2.62 to $+1.0 \mathrm{~min}$. There was a similar reduction in blood pressure and OSArelated costs in both groups. This study highlights the 
premise that auto-CPAP and fixed CPAP are equivalent within prespecified ranges in improving objective and subjective sleepiness in subjects with OSA over the course of 2 years.

The type of mask interface used (ie, nasal or oronasal) is an important consideration in the management of an individual requiring PAP therapy. The overall effects of either therapy on PAP adherence are unclear. A metaanalysis by Andrade et $\mathrm{al}^{9}$ evaluated the residual AHI and CPAP adherence in the treatment of OSA between nasal versus oronasal masks. This meta-analysis included studies comparing nasal and oral CPAP for OSA (ie, an original article and randomized and nonrandomized trials). Five randomized and nonrandomized trials $(N=5,633$ subjects) were identified. The random effects meta-analysis revealed that oronasal masks, as compared to nasal masks, were associated with a significantly higher CPAP level, higher residual AHI, and a poorer adherence rate.

\section{Oral-Appliance Therapy}

Oral-appliance therapy for the treatment of OSA is being more frequently used as either an alternative to or an adjunct treatment to PAP therapy. Compared with the accepted standard (ie, CPAP therapy), oral appliances are less efficacious but are more accepted and better tolerated. This may in turn lead to comparable or even increased levels of therapeutic effectiveness. Hamoda et al ${ }^{10}$ provided an update on the current literature in the field and discussed various types of oral appliances available, indications for their use, and the process for device titration. The effectiveness of both CPAP and oral-appliance therapy, with an emphasis on reduction of the AHI was compared. It is important to recognize that merely using the AHI to demonstrate therapeutic effectiveness is oversimplified, and that one must take into consideration the hours per night the treatment is physically used and whether the treatment is being used while the patient is asleep. A description of the use of combined therapy (ie, CPAP plus oral appliance therapy, or positional therapy plus oral appliance therapy) has reported that the combination may be beneficial for certain patients. Finally, the potential shortterm side effects of oral-appliance therapy (eg, increased mouth dryness or salivation, teeth and gum pain or discomfort, a perception of an abnormal occlusion in the morning, muscle tenderness, and jaw stiffness in the shortterm) were noted. Longer-term side effects included predominantly dental and occlusal changes.

\section{Surgical Therapy}

Bariatric Surgical Guidelines. Bariatric surgical procedures are increasing in number. The prevalence of OSA is very high (at times $>60 \%$ ) in this population. de Raaff and colleagues ${ }^{11}$ provided a consensus guideline on the perioperative management of OSA and bariatric surgery. All subjects undergoing bariatric surgery should be screened for OSA and obesity hypoventilation syndrome. CPAP in the first treatment choice for moderate to severe OSA, and bi-level PAP or supplemental oxygen should be used in cases of inadequate ventilation or oxygen desaturation, respectively. Intra-operative approaches include preoxygenation, ramped position, CPAP or PAP during induction, supplemental high-flow oxygen, and low tidal volume. Avoidance of opioids should be attempted during the postoperative period. CPAP in the immediate postoperative period reduces the AHI and improves oxygen saturation. Continuous assessment by monitoring vital signs and oxygen saturation (with a minimum of pulse oximetry) should be continued in the postoperative period until the patient is no longer at risk for respiratory depression.

Neurostimulation Surgery. Surgical therapy for OSA has continued to evolve over the last 3 decades. The use of neurostimulation therapy (eg, upper airway stimulation via a unilateral hypoglossal nerve implantation) has gained increased interest in the field as an alternative treatment option for those unable to tolerate CPAP for the treatment of OSA. A generalized review of this modality, including the foundation for such therapy, the conceptual development of the procedure, subject selection criteria, and results of the clinical research studies, was recently published. ${ }^{12}$

The results of the ADHERE Registry, which includes both retrospective and prospective trials, included 301 subjects with moderate to severe OSA and intolerance to CPAP who underwent upper airway stimulation surgery. The mean AHI decreased from $35.6 \pm 15.3$ to $10.2 \pm 12.9$ events/h $(P<.001)$, and Epworth Sleepiness Scale scores decreased from $11.9 \pm 5.5$ to $7.5 \pm 4.7(P<.001)$. Very few complications occurred, and $90 \%$ of the subjects were more satisfied with upper airway stimulation than with CPAP. ${ }^{13}$ The 5-y outcomes from a prospective cohort of subjects with OSA who underwent upper airway stimulation via a unilateral hypoglossal nerve implant in a multi-center study were published. Improvement in sleepiness and quality of life were observed. AHI response rate in 71 subjects (defined as an AHI $<20$ events/h and $>50 \%$ reduction) was $75 \%$. The responder rate was $63 \%$ at 5 years. Serious device-related events were noted in $6 \%$ of subjects. ${ }^{14}$

\section{Pharmacologic Therapy}

The treatment for OSA currently includes PAP, oral appliances, and surgical therapies. There is no effective pharmacologic treatment. A study involving 20 subjects 
with an elevated AHI was performed to determine the effects of the combination of a norepinephrine reuptake inhibitor (ie, atomoxetine) and an anti-muscarinic agent (ie, oxybutynin) on OSA severity and genioglossus responsiveness in subjects with an elevated AHI. ${ }^{15}$

The subjects (median age $53 \mathrm{y}$, body mass index 34.8 $\mathrm{kg} / \mathrm{m}^{2}$ ) were randomized in a placebo-controlled, doubleblind, crossover trial comparing 1 night of atomoxetine $(80 \mathrm{mg}$ ) plus oxybutynin $(5 \mathrm{mg})$ to placebo administered prior to sleep. AHI and genioglossus muscle responsiveness were measured. A lowering of the AHI by $63 \%$ (range, $34-86 \%$ ), from 28.5 (range, 10.9-51.6) to 7.5 (range, 2.4-18.6) events per hour $(P<.001)$ was noted in the group that received the combination therapy. Neither drug alone was shown to reduce the AHI. Genioglossus muscle responsiveness improved in the group that received combination therapy. While further research in the area is needed, these findings open new possibilities for the pharmacologic treatment of OSA.

\section{Exercise and Rehabilitation}

OSA is highly prevalent in individuals with heart failure. Although exercise training for those with heart failure has been reported to improve cardiovascular function, functional capacity, and quality of life, its effects on OSA is unknown. Hupin et a ${ }^{16}$ performed a study to evaluate the effects of exercise on OSA for those subjects with heart failure and a reduced ejection fraction. A total of $65 \mathrm{sub}-$ jects were randomized to one of 4 groups: control, exercise only, CPAP only, and exercise plus CPAP. The mean AHI did not change significantly in the control group, whereas it decreased moderately in the exercise group and decreased significantly more in the CPAP group and in the exercise plus CPAP groups. The combination of exercise and CPAP improved subjective excessive daytime somnolence, quality of life, and New York Heart Association functional class. Exercise alone attenuated OSA and improved quality of life more than CPAP alone. These results highlight the importance of the appropriate therapy in patients with heart failure, reduced ejection fraction, and OSA, and they also emphasize the role of exercise training for this population.

Regular physical activity improves OSA in the general population. This finding, however, has not been assessed in patients who have suffered a myocardial infarction and are enrolled in cardiac rehabilitation. Servantes et al ${ }^{17}$ studied 105 subjects with known coronary artery disease and concomitant OSA to determine the effects of cardiac rehabilitation on the AHI. Following the completion of cardiac rehabilitation, the AHI, determined from electocardiogram-derived respiratoin obtained from nocturnal Holter electrocardiographic recordings, decreased significanlty $(-9.3 \pm 9.5, P<.001)$ for subjects with severe OSA. The decrease was even greater when peak oxygen consumption and baroreflex sensitivity improved beyond 20\% compared to baseline values $(-11.6 \pm 9.1, P<.001)$. Severe OSA in subjects with coronary artery disease was significantly improved after 2 months of cardiopulmonary rehabilitation. The authors felt that changes in autonomic nervous system activity through physical activity might be a target for complementary therapy of OSA in patients with coronary artery disease. ${ }^{17}$

\section{Therapy Adherence}

\section{Hospital Readmissions and Adherence}

Hospital readmissions have gained significant attention from care providers and third-party payers. Strategies to help reduce such readmissions are being undertaken by many health care teams. The impact of sleep disorders (and their treatment) on readmission rates has yet to be studied. The primary objective of a study by Truong et al ${ }^{18}$ was to determine whether nonadherence to CPAP was associated with an increased all-cause, cardiovascularcause, or pulmonary-cause 30-d hospital readmission rate. This retrospective cohort study conducted at a Veterans Affairs hospital included subjects who had OSA and were hospitalized over an 8-y period ending in 2015. The authors reported a significantly higher ratio of 30-d all-cause readmission in 345 subjects (183 adherent and 162 not adherent to CPAP therapy) in the nonadherent group with an adjusted odds ratio of 3.52 (95\% CI 2.04-6.08, $P<.001)$. The $30-\mathrm{d}$ readmission rate with a cardiovascular cause was significantly higher in the nonadherent group as well.

\section{Patient-Engagement Technologies}

Effective sleep apnea treatment is often suboptimal due to a lack of patient adherence to the prescribed therapy. Investigators compared CPAP therapy adherence among subjects who were provided technology that allowed for active patient engagement versus those who received usual care monitoring. ${ }^{19}$ Adherence data from 2 cloud-based databases were analyzed for subjects with OSA $(N=$ $128,037)$. The active patient engagement group had more subjects achieving adherence criteria $(87.3 \%)$ compared with usual care monitoring group $(70.4 \%, P<.001$ for the difference). The average nightly therapy usage was $5.9 \mathrm{~h}$ in the active patient engagement group versus $4.9 \mathrm{~h}$ in the usual care monitoring group $(P<.001)$. This study postulates that improved adherence rates can be achieved by adding modern technology to usual care when treating patients with OSA. Randomized controlled trials are needed to further define this relationship. 


\section{Other Publications}

\section{Medical Cannabis}

Medical cannabis is becoming more widely used for a variety of medical conditions. It has been postulated that this therapy may provide benefit for individuals with OSA. The composition of cannabinoids within medical cannabis vary significantly and are not regulated. Dronabinol is not cleared by the FDA for the treatment of OSA, and its long-term effects (and safety) on sleep quality are unknown. Many subjects have reported somnolence as a shortterm side effect. It is the position of the AASM that medical cannabis and its synthetic extracts should not be used for the treatment of OSA due to unreliable delivery methods and insufficient evidence of effectiveness, tolerability, and safety. ${ }^{20}$

\section{Personalized Management}

The phenotypic traits associated with OSA include anatomic and nonanatomic components. The contribution of these traits varies among individuals. Current therapy often targets anatomical components: CPAP, oral appliances, weight loss, and upper airway surgery. Nonanatomic contributors include a low respiratory arousal threshold, ineffective or reduced pharyngeal dilator muscle activity, and unstable ventilatory control, and these components are prompting research into newer therapeutic targets (eg, pharmacologic agents, hypoglossal nerve stimulation). Identification of a particular individual's phenotypic traits may help develop targeted therapy and thus help personalize the management of individual patients. ${ }^{21}$

\section{Reflections on Nasal CPAP and OSA}

CPAP therapy has been used for OSA for more than 4 decades. In 1980, sleep apnea was considered a rare disorder and, even after the successful initial treatment experiments with CPAP therapy, the extent of OSA across the spectrum of medical illness was not appreciated. Sullivan ${ }^{22}$ reflected on the inspiration behind the idea of CPAP, providing a description of the first CPAP experiment as well as information on the extension of CPAP from the bench setting to routine clinical use and then to the development of noninvasive nasal ventilation. Sullivan's article is a must-read for those interested in the history of PAP therapy as it is used for the treatment of OSA. ${ }^{22}$

\section{Summary}

Over the last year, there has been a significant increase in the amount of research in the field of sleep-disordered breathing. Research into the associated comorbid condi- tions (eg, heart failure, coronary artery disease, depression) and treatment of the underlying OSA has increased. The benefits of therapies (eg, PAP therapy, oral appliance therapy, automatically adjusted CPAP therapy, neurostimulation surgical therapies, and even pharmacologic therapy) have been noted. Adherence to CPAP therapy is reported to be associated with reduced rehospitalization rates, and newer technologies are being developed to help patients increase their adherence to PAP. Finally, several new guidelines and position statements have been issued by the AASM (eg, home sleep apnea test, medical cannabis, consumerbased technologies) to help clarify the position of various modalities in the overall diagnosis and treatment of OSA. This research and these position statements will help us develop more effective and personalized diagnostic and therapeutic plans for patients who have sleep-disordered breathing.

\section{REFERENCES}

1. Kendzerska T, Leung RS, Aaron SD, Ayas N, Sandoz JS, Gershon AS; for the Canadian Respiratory Research Network. Cardiovascular outcomes and all-cause mortality in patients with obstructive sleep apnea and chronic obstructive pulmonary disease (overlap syndrome). Ann Am Thorac Soc 2019;16(1):71-81.

2. Balcan B, Thunstrom E, Strollo PJ, Peker Y. CPAP treatment and depression in adults with coronary artery disease and nonsleepy obstructive sleep apnea: a secondary analysis of the RICCADSA trial. Ann Am Thorc Soc 2019;16(1):62-70.

3. Suen C, Ryan CM, Mubashir T, Ayas NT, Abrahamyan L, Wong J, et al. Sleep study and oximetry parameters for predicting postoperative complications in patients with OSA. Chest 2019;155(4):855867.

4. Rosen IM, Kirsch DB, Chervin RD, Carden KA, Ramar K, Aurora $\mathrm{N}$, et al. Clinical use of a home sleep apnea test: an American Academy of Sleep Medicine position statement. J Clin Sleep Med 2017;13(10):1205-1207.

5. Aurora RN, Patil SP, Punjabi NM. Portable sleep monitoring for diagnosing sleep apnea in hospitalized patients with heart failure. Chest 2018;154(1):91-98.

6. Khosla S, Deak MD, Gault D, Goldstein CA, Hwang D, Kwon Y, et al. Consumer sleep technology: an American Academy of Sleep Medicine position statement. J Clin Sleep Med 2018;14(5):877-880.

7. Billings ME, Krishnan V, Su G, Donovan LM, Patel SR, Hudgel DW, et al. Summary for clinicians: the role of weight management in the treatment of adult obstructive sleep apnea. Ann Am Thorac Soc 2019;16(4):405-408.

8. Bloch KE, Huber F, Furian M, Latshang TD, LoCascio CM, Nussbaumer-Ochsner Y, et al. Autoadjusted versus fixed CPAP for obstructive sleep apnoea: a multicenter, randomized equivalence trial. Thorax 2018;73(2):174-184.

9. Andrade RGS, Viana FM, Nascimentio JA, Drager LF, A Moffa, Brunoni AH, et al. Nasal vs oronasal CPAP for OSA treatment: a meta-analysis. Chest 2018;153(3):665-674.

10. Hamoda MH, Kohzuka Y, Almeida FR. Oral appliances for the management of OSA: an updated review of the literature. Chest 2018;153(2):544-553.

11. de Raaff CAL, de Vries N, van Wagensveld BA. Obstrutive sleep apnea and bariatric surgical guidelines: summary and update. Curr Opin Anesthesiol 2018;31(1):104-108. 


\section{YeAR IN ReVIEW: SLeEP}

12. Curado TF, Oliven A, Sennes LU, Polotsky VY, Eisele D, Schwartz AR. Neurostimulation treatment of OSA. Chest 2018;154(6):14351447.

13. Boon M, Huntley C, Steffen A, Maurer JT, Sommer JU, Schwab R, et al. Upper airway stimulation for obstructive sleep apnea: results from the ADHERE registry. Otolaryngol Head Neck Surg 2018; 159(2):379-385.

14. Woodson BT, Strohl KP, Soose RJ, Gillespie MB, Maurer JT, de Vries N, et al. Upper airway stimulation for obstructive sleep apnea: 5-year outcomes. Otolaryngol Head Neck Surg 2018;159(1):194202.

15. Taranto-Montemurro L, Messineo L, Sands SA, Azarbarzin, Marques M, Edwards BA, et al. The combination of atomoxetine and oxybutynin greatly reduces obstructive sleep apnea severity: a randomized, placebo-controlled, double-blind crossover trial. Am J Respir Crit Care Med 2019;199(10):1267-1276.

16. Hupin D, Pichot V, Berger M, Sforza E, Raffin J, Lietar D, et al. Obstructive sleep apnea in cardiac rehabilitation patients. J Clin Sleep Med 2018;14(7):1119-1126.
17. Servantes DM, Javaheri S, Kravchychyn ACP, Storti LJ, Almeida DR, de Mello MT, et al. Effects of exercise training and CPAP in patients with heart failure and OSA. Chest 2018;154(4):808-817.

18. Truong KT, De Jardin R, Massoudi N, Hashemzadeh M, Jafari B. Nonadherence to CPAP associated with increased 30-day hospital readmissions. J Clin Sleep Med 2018;14(2):183-189.

19. Malhotra A, Crocker ME, Willes L, Kelly C, Lynch S, Benjafield AV. Patient engagement using new technology to improve adherence to positive airway pressure therapy: a retrospective analysis. Chest 2018;153(4):843-850.

20. Ramar K, Rosen IM, Kirsch DB, Chervin RD, Carden KA, Aurora $\mathrm{RN}$, et al. Medical cannabis and the treatment of obstructive sleep apnea: an American Academy of Sleep Medicine position statement. J Clin Sleep Med 2018;14(4):679-681.

21. Carberry JC, Amatoury J, Eckert DJ. Personalized management approach for OSA. Chest 2018;153(3):744-755.

22. Sullivan CE. Nasal positive airway pressure and sleep apnea: reflections on an experimental method that became a therapy. Am J Respir Crit Care Med 2018;198(5):581-587. 